\title{
ORIGINAL ARTICLE Individual spatial aggregation correlates with between- population variation in fine-scale genetic structure of Silene ciliata (Caryophyllaceae)
}

\author{
C Lara-Romeroํ, A García-Fernández ${ }^{1}$, JJ Robledo-Arnuncioº ${ }^{2}$ M Roumet ${ }^{3}$, J Morente-López ${ }^{1}$, A López-Gil ${ }^{1}$ \\ and JM Iriondo ${ }^{1}$
}

Fine-scale genetic structure (FSGS) can vary among populations within species depending on multiple demographic and environmental factors. Theoretical models predict that FSGS should decrease in high-density populations and increase in populations where individuals are spatially aggregated. However, few empirical studies have compared FSGS between populations with different degrees of individual spatial aggregation and microhabitat heterogeneity. In this work, we studied the relationship between spatial and genetic structure in five populations of alpine specialist Silene ciliata Poiret (Caryophyllaceae). We mapped all individuals in each population and genotyped 96 of them using 10 microsatellite markers. We found significant FSGS consistent with an isolation-by-distance process in three of the five populations. The intensity of FSGS was positively associated with individual spatial aggregation. However, no association was found between FSGS and global population density or microhabitat heterogeneity. Overall, our results support theoretical studies indicating that stronger spatial aggregation tends to increase the magnitude of FSGS. They also highlight the relevance of characterizing local plant distribution and microhabitat to better understand the mechanisms that generate intraspecific variation in FSGS across landscapes.

Heredity (2016) 116, 417-423; doi:10.1038/hdy.2015.102; published online 25 November 2015

\section{INTRODUCTION}

Fine-scale spatial genetic structure (FSGS) is defined as the nonrandom spatial distribution of genotypes within plant populations. This pattern influences evolutionary parameters and processes like biparental inbreeding, kin competition, effective population size and local adaptation that in turn affect individual and population fitness (Linhart and Grant, 1996; Leblois et al., 2006). FSGS results from the complex interaction between limited gene flow through seed and pollen dispersal, genetic drift and local selection (Ennos, 1994; Vekemans and Hardy, 2004). Restricted gene dispersal in a continuous plant population theoretically leads to local inbreeding and the build-up of family structures across generations. In the absence of homogenizing selection, this phenomenon is expected to result in the nonrandom spatial organization of genotypes or FSGS (Ennos, 1994; Vekemans and Hardy, 2004).

A common form of FSGS is genetic isolation by distance (IBD), that is, a positive relationship between genetic and geographic distances among individuals (Wright, 1943). IBD patterns are usually measured by the rate of decrease in the probability of gene identity with spatial distance, which has been shown to be determined by the product of the effective gene dispersal rate, $\sigma_{\mathrm{e}}$, and effective population density, $D_{\mathrm{e}}$ ('neighbourhood size' $N b$, sensu Wright, 1943; Rousset, 2000).
As $\sigma_{\mathrm{e}}$ and $D_{\mathrm{e}}$ can vary among species and populations, FSGS patterns should show variation at both hierarchical levels.

Most empirical studies on plant FSGS patterns have focused on the influence of interspecific life-history variation, and particularly on the IBD patterns of species with different life forms, mating systems, and seed and pollen dispersal strategies (Vekemans and Hardy, 2004; Hardy et al., 2006; Hamrick and Trapnell, 2011). The determinants of intraspecific variation in FSGS remain comparatively underexplored, although intraspecific competition, microenvironmental selection and historic events such as founder effects or other demographic disturbances have been cited (Linhart et al., 1981; Tero et al., 2005; Chung et al., 2007). Differences in FSGS among populations may partly arise from variation in population density and spatial aggregation of individuals generated by the above-mentioned determinants. The magnitude of FSGS is expected to be higher in low-density populations characterized by a low $D_{\mathrm{e}}$ (Leblois et al., 2004; Vekemans and Hardy, 2004). In addition, for a given population density, several numerical simulation studies have shown that the spatial aggregation of adult plants generally increases the magnitude of FSGS compared with random or regular distributions (Doligez et al., 1998; Leblois et al., 2004; Robledo-Arnuncio and Rousset, 2010). Recent empirical studies have also shown that plant populations in fragmented habitats exhibit stronger FSGS than in continuous habitats (see, for example,

${ }^{1}$ Biodiversity and Conservation Area, School of Experimental Sciences (ESCET), Universidad Rey Juan Carlos, Madrid, Spain; ${ }^{2}$ Department of Forest Ecology and Genetics, INIA-CIFOR, Madrid, Spain and ${ }^{3}$ Plant Ecological Genetics, Institute of Integrative Biology (IBZ), ETH Zurich, Zurich, Switzerland

Correspondence: Dr C Lara-Romero, Biodiversity and Conservation Area, School of Experimental Sciences (ESCET), Universidad Rey Juan Carlos, C/Tulipán s/n, E-28933 Móstoles, Madrid, Spain.

E-mail: carlos.lara.romero@gmail.com

Received 1 May 2015; revised 29 September 2015; accepted 13 October 2015; published online 25 November 2015 
Valbuena-Carabaña et al., 2007; Wang et al., 2011; but see Born et al., 2008). However, empirical studies examining variation in FSGS among populations with different densities and degrees of spatial aggregation remain scarce (Hamrick and Trapnell, 2011), and FSGS has not been analysed together with the spatial distribution of individuals and microhabitat heterogeneity (but see Chung et al., 2007; Jacquemyn et al., 2009; Van Heerwaarden et al., 2010). Further comparative studies of FSGS across populations with different demographic and microhabitat characteristics are needed to better understand the mechanisms generating intraspecific variation in FSGS across different landscapes (see, for example, Born et al., 2008; Wang et al., 2011).

The alpine specialist Silene ciliata Poiret (Caryophyllaceae) constitutes a suitable system to study the demographic and environmental determinants of intraspecific variation in FSGS. The low effective seed dispersal distance of this species (Lara-Romero et al., 2014) and the possibility of selfing (García-Fernández et al., 2012) may yield substantial FSGS. Previous studies have shown strong variation in demographic structure (for example, density and spatial aggregation of individuals) and physical environment (for example, microhabitat heterogeneity and water availability) among populations (GimenezBenavides et al., 2007, 2008; Lara-Romero et al., 2014). In this study, we described the spatial genetic structures of five demographically stable but spatially heterogeneous populations of $S$. ciliata and assessed whether FSGS intensity increases in populations with low plant density and high spatial aggregation. We addressed three main questions: (1) Do S. ciliata populations have FSGS? (2) Does the intensity of FSGS vary among populations? (3) If so, is this variation associated with differences in demographic structure (that is, population density and individual spatial aggregation) and/or microhabitat heterogeneity?

\section{MATERIALS AND METHODS}

\section{Study region and species}

S. ciliata Poiret (Caryophyllaceae) is a perennial cushion plant that occurs in the Mediterranean mountain ranges of southern Europe. It is self-compatible, although autogamy is restricted by pronounced protandry (García-Fernández et al., 2012). It blooms in late summer (Gimenez-Benavides et al., 2007) and its flowering stems reach $15 \mathrm{~cm}$ in height and have 1-5 flowers. S. ciliata is pollinated at night by Hadena consparcatoides Schawerda (Lepidoptera, Noctuidae) but can also be pollinated by diurnal insects (Gimenez-Benavides et al., 2007). Fruit capsules contain up to 100 seeds that are wind dispersed in August-September. Average effective seed dispersal distances are relatively invariant across populations (mean \pm s.e.: $0.40 \pm 0.08 \mathrm{~m}$, Lara-Romero et al., 2014).

This study was carried out in the Sierra de Guadarrama, a mountain range located in central Spain. S. ciliata grows locally at altitudes from 1900 to $2430 \mathrm{~m}$ in dry cryophilic pastures dominated by Festuca curvifolia Lag ex Lange and other perennial plants that are interspersed in a shrub matrix characterized by Cytisus oromediterraneus Rivas Mart. et al. and Juniperus communis subsp. alpina (Suter) Čelak. Mean annual precipitation $\left(40^{\circ} 46^{\prime} \mathrm{N}, 4^{\circ} 19^{\prime} \mathrm{W} ; 1860 \mathrm{~m}\right.$ above sea level) is $1330 \mathrm{~mm}$ with a pronounced dry season $(<10 \%$ of total annual rainfall) from May to October. Mean annual temperature is $6.3^{\circ} \mathrm{C}$, with mean monthly temperatures ranging from $-1{ }^{\circ} \mathrm{C}$ in January to $16^{\circ} \mathrm{C}$ in July (www.aemet.es). Although S. ciliata presents variable ploidy levels in natural populations, all individuals from the studied Guadarrama populations are diploid (2n =24, García-Fernández et al., 2012).

\section{Study plots}

In August and September 2010, we established one $10 \times 10 \mathrm{~m}$ plot in each of five $S$. ciliata populations distributed along the Sierra de Guadarrama (Figure 1a and Table 1). Additional information on the selected populations can be found in Lara-Romero et al., 2014. Based on the low human intervention in the area and long-term demographic monitoring of two of the populations (Peñalara (PEN) and Laguna (LAG)), we expected these populations to be at equilibrium. Genetic tests for detecting past changes in effective population size support the demographic stability of the populations (Appendix 1 in Supplementary Information). We mapped all adult $S$. ciliata individuals in each study plot using two high-resolution Differential Global Navigation Satellite System (DGNSS) receivers (Viva GS15, Leica, Switzerland) with an absolute accuracy of $5 \mathrm{~cm}$ for $x$ and $y$ coordinates. We also mapped the spatial position and contour of all neighbouring C. oromediterraneus and J. communis shrubs, rocky outcrops and rocks $>50 \mathrm{~cm}$ in diameter that are unsuitable microhabitats for S. ciliata (Figure 1a). We then used this information to estimate global density $(D)$ and to characterize the spatial distribution of the adult plants in each study plot (that is, O-ring statistic, see below), as well as microhabitat heterogeneity, calculated with the shape index $(S I)$. SI is widely used in landscape ecology as a measure of landscape complexity and fragmentation (Berry, 2007). It has a value of one when the patch is a perfect circle/square and increases as the patch becomes more irregular (Berry, 2007). The five populations spanned a gradient in adult density $(D)$ and had different degrees of microhabitat heterogeneity (Table 1 ).

\section{DNA extraction and microsatellite genotyping}

We collected leaf material from $\sim 96$ adult plants per plot for genetic analysis (Table 1). To increase the sample size of shorter interindividual distances for autocorrelation analysis, plants were selected using a clustered random sampling design (sensu Storfer et al., 2007). Namely, we randomly selected 24 sample locations in each plot, where we collected leaf material from four adult individuals located in close proximity to one other. The minimum sample distance ranged between $10 \mathrm{~cm}$ (Nevero (NEV)) and $28 \mathrm{~cm}$ (Cabezas de Hierro $(\mathrm{CAB})$ ). We collected large individuals of similar rosette size to prevent sampling individuals belonging to different age classes (that is, parents and offspring).

DNA was extracted using the DNeasy Plant minikit (QIAGEN, Valencia, CA, USA) with 10-20 mg of dried S. ciliata tissue. Ten microsatellite loci previously used in genetic studies of $S$. ciliata were selected for genotyping: Sci1224, Sci1208, Sci0106, Sci1443, EST-2HTS, EST-8HTS, EST-37HTS, EST-X4-3, EST-G34D06 and EST-G47A02 (García-Fernández et al., 2012). Specifications of PCR reactions and amplification are described in García-Fernández et al. (2012). Samples were run on an automated DNA sequencer (ABI PRISM 3730, Applied Biosystems, Foster City, CA, USA) in Parque Científico de Madrid (Madrid, Spain). Fragment size was determined using GeneMarker version 1.85 (SoftGenetics, State College, PA, USA).

We evaluated genotyping accuracy by re-amplifying and re-scoring a random subset of samples. We also estimated the occurrence of null alleles, large-allele dropout and stuttering using the software MICRO-CHECKER (Van Oosterhout et al., 2004). We discarded loci Scil443 and EST-SSR X4-3 because of inconsistent allelic scoring. Locus Sci1224 showed signs of potential null alleles because of an excess of homozygotes in some allele size classes. Subsequent analysis produced the same results and conclusions irrespective of whether or not this locus was included, except for the fixation index $\left(F_{I S}\right)$. Therefore, locus Sci1224 was excluded from the calculation of $F_{\text {IS }}$ (see below). Selected loci were found to be statically independent $(P>0.05$ : Log-likelihood ratio G statistic based on 5000 permutations performed in GENEPOP v. 4.2, http://genepop.curtin.edu.au/).

\section{Spatial distribution of adult plants within populations}

We employed the noncumulative O-ring statistic $\mathrm{O}(r)$ (Wiegand and Moloney, 2004) to characterize the spatial distribution of adult plants in the five study populations. $\mathrm{O}(r)$ gives the expected number of points (that is, plants) in a ring at distance $r$ from an arbitrary point (Wiegand and Moloney, 2004). We calculated $\mathrm{O}(r)$ considering concentric rings of a constant width of $0.1 \mathrm{~m}$. To avoid estimation bias, $\mathrm{O}(r)$ was calculated between $r=0.10$ and $2.5 \mathrm{~m}$, that is, up to a quarter of plot width (Baddeley and Turner, 2005). We used Ripley's isotropic correction (Baddeley and Turner, 2005) to correct for edge effects. To assess the existence of significant spatial aggregation within populations, we generated $95 \%$ simulation envelopes associated to the null model of complete spatial randomness using the tenth lowest and tenth highest value of 399 Monte Carlo simulations of the complete spatial randomness-null model. $\mathrm{O}(r)$ values above and below the envelope indicate significant spatial aggregation and repulsion compared with a complete spatial randomness pattern, respectively (Wiegand and Moloney, 2004). 

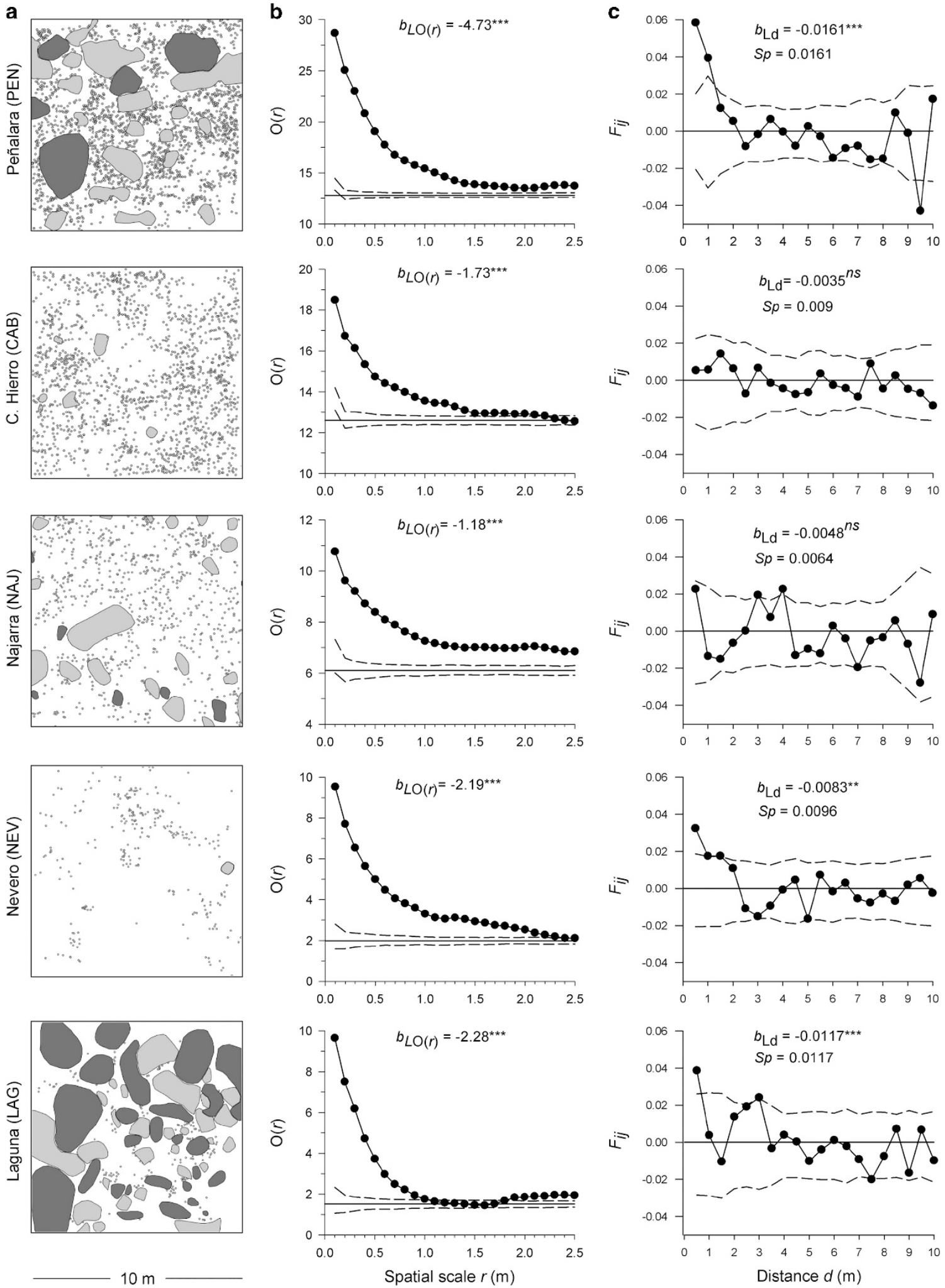

Figure 1 Spatial distribution of adult plants (a), spatial demographic structure (b) and spatial genetic structure (c) in Silene ciliata study plots. (a) Spatial distribution of adult plants (small circles), rocks (grey) and shrubs (dark grey) in each study plot of $S$. ciliata. (b) Spatial demographic structure: $b_{\text {LO(r) }}$ denotes the slope of the regression of the $O$ ring statistic, $O_{(r)}$ on the logarithm of the spatial distance, $\ln (r)$; the thin solid line indicates average intensity of the point pattern (that is, plant density) and dashed lines indicate 95\% confidence envelopes under the null hypothesis of random spatial structure. Note that $y$ axes show different scales for clarity. (c) Spatial genetic structure: $b_{\mathrm{Ld}}$ denotes the slope of the regression of kinship coefficient values $\left(F_{\mathrm{ij}}\right)$ on the logarithm of the spatial distance, In $\left(d_{i j}\right)$; dashed lines represent 95\% confidence envelopes constructed under the null hypothesis (no genetic autocorrelation exists). ${ }^{* *} P<0.01,{ }^{* * *} P<0.001$ 
Table 1 Description of sampling sites and genetic diversity estimates in Silene ciliata populations

\begin{tabular}{|c|c|c|c|c|c|c|c|c|c|c|c|c|}
\hline$\frac{\text { Population }}{\text { Peñalara }}$ & $\begin{array}{l}\text { Abbreviation } \\
\text { PEN }\end{array}$ & \multicolumn{2}{|c|}{ UTM coordinates ( $m$ ) } & $\frac{D}{12.8}$ & $\frac{D_{\mathrm{e}}}{3.57}$ & $\frac{S H(\%)}{71.3}$ & $\frac{S I}{1.91}$ & $\frac{N}{96}$ & $\frac{A}{10}$ & $\begin{array}{c}H_{0} \\
0.39\end{array}$ & $\frac{H_{\mathrm{E}}}{0.50}$ & $\frac{F_{\mathrm{IS}}}{0.15^{*}}$ \\
\hline Cabezas de Hierro & $\mathrm{CAB}$ & 421169 & 4516859 & 12.6 & 3.34 & 96.8 & 1.14 & 94 & 10 & 0.41 & 0.55 & $0.23 *$ \\
\hline Nevero & NEV & 428863 & 4537167 & 1.99 & 0.54 & 99 & 1.11 & 96 & 12 & 0.39 & 0.55 & $0.19 *$ \\
\hline Laguna & LAG & 419931 & 4521082 & 1.52 & 0.40 & 48.8 & 2.40 & 93 & 11 & 0.34 & 0.53 & $0.26^{*}$ \\
\hline
\end{tabular}

Abbreviations: $A$, average number of alleles per locus; $D$, census plant density (individuals per $\mathrm{m}^{2}$ ); $D_{\mathrm{e}}$, effective plant density; $F_{\mathrm{IS}}$, average inbreeding coefficient; $H_{0}$ and $H_{\mathrm{E}}$, expected and observed heterozygosities; N, sample size; SH, proportion of suitable habitat; SI, shape index of suitable habitat; UTM, $x, y$ geographical coordinates.

Significance level: ${ }^{*} P<0.001$.

Finally, to compare the overall strength of aggregation among populations, we calculated the slope $\left(b_{\mathrm{LO}(r)}\right)$ of the linear regression of $\mathrm{O}(r)$ on the logarithm of the spatial distance $r$ for each population. Associated s.d. was also estimated and used to perform pairwise $t$-tests to determine whether $b_{\mathrm{LO}(r)}$ significantly differed among populations. All statistical analyses were performed in the open source software R v 3.0.2 using the package 'spatstats' (Baddeley and Turner, 2005).

\section{Population genetic analyses}

We used GENEPOP to calculate standard population genetic measures, including total number of alleles $(A)$, observed $\left(H_{\mathrm{O}}\right)$ and expected $\left(H_{\mathrm{E}}\right)$ heterozygosity, and inbreeding coefficients $\left(F_{\mathrm{IS}}\right)$. We used per-locus values to test for statistical differences in genetic measures across populations using the nonparametric Friedman rank sum test. Deviations from Hardy-Weinberg equilibrium within each population were tested using Fisher's exact test implemented in GENEPOP.

\section{Characterization of FSGS}

Bayesian clustering analysis. Within-population spatial heterogeneity could limit gene movement between individuals separated by physical obstacles (for example, shrub, rock and so on) or growing in a different environment (that is, gene flow is limited because of selection acting against locally maladapted migrant genes and individuals) (Storfer et al., 2007). When abiotic and/or biotic discontinuities remain static across generations, genetic diversity could be geographically clustered in each plot. To address this possibility, we used a Bayesian clustering method implemented in the R package 'Geneland' version 4.0.3 (Guillot et al., 2005). This approach uses Markov chain Monte Carlo procedures to estimate the number of genetic clusters that is treated as an unknown parameter. Five independent runs of the spatial $D$-model were performed with 100000 Markov chain Monte Carlo iterations, of which every hundredth one was saved.

Spatial genetic autocorrelation analysis. To assess FSGS resulting from IBD, we used the spatial autocorrelation approach implemented in SPAGeDi version 1.4 (Hardy and Vekemans, 2002). In each population, we estimated the genetic similarity between every pair of individuals $i$ and $j$ with Loiselle's kinship coefficient ( $F_{\mathrm{ij}}$, Loiselle et al., 1995). We then regressed the obtained values on the spatial distance between individuals and its natural logarithm $\left(d_{\mathrm{ij}}\right.$ and $\ln$ $\left(d_{\mathrm{ij}}\right)$, respectively) and reported the regression slopes ( $b_{\mathrm{d}}$ and $b_{\mathrm{Ld}}$, respectively) for each type of regression. The s.e. values for $F_{\mathrm{ij}}, b_{\mathrm{d}}$ and $b_{\mathrm{Ld}}$ were estimated by jackknifing over loci. We addressed the significance of the spatial variation of $F_{\mathrm{ij}}$ by computing the average pairwise $F_{\mathrm{ij}}$ over a set of distance intervals $(d)$ comprising between 0.5 and $10 \mathrm{~m}$ and comparing these values with those expected under the null hypothesis of absence of IBD (that is, $F_{\mathrm{ij}}$ and $d_{\mathrm{ij}}$ or $\ln \left(d_{\mathrm{ij}}\right)$ are uncorrelated). The confidence interval associated to the null hypothesis was constructed by performing 9999 random permutations of genotypes among spatial positions.

To compare FSGS intensity among populations, we calculated the statistic $S p$ for each population (Vekemans and Hardy, 2004). We used the formula $S p=-b_{\mathrm{Ld}} /\left(1-F_{1}\right)$, where $F_{1}$ is mean $F_{\mathrm{ij}}$ at the smallest distance interval $d$. Mean $S p$ and s.e. were calculated for each population over all loci, and the approximate $95 \%$ confidence intervals (CIs) were obtained as \pm 1.96 times the s.e. estimates obtained across loci. As before for $b_{\mathrm{LO}(\mathrm{r})}$, we carried out pairwise $t$-tests to determine the statistical significance of the differences found between populations.

We also used the software SPAGeDi to estimate Wright's neighbourhood size $\left(N b\right.$, Wright, 1943) and effective gene dispersal rate $\left(\sigma_{e}\right)$ of each population.

Under Wright's IBD model, $\mathrm{Nb}$ can be estimated in two-dimensional populations as (Vekemans and Hardy, 2004):

$$
N b=-\left(1-F_{1}\right) / b_{r},
$$

where $b_{\mathrm{r}}$ is the restricted regression slope $\left(b_{\mathrm{Ld}}\right)$ of $F_{\mathrm{ij}}$ on $\ln \left(d_{\mathrm{ij}}\right)$ in the range $\sigma_{\mathrm{e}}<\mathrm{d}<20 \quad \sigma_{\mathrm{e}}$. The expected relationship between $\mathrm{Nb}$ and $\sigma_{\mathrm{e}}$ is given by (Rousset, 2000):

$$
N b=4 \pi D_{\mathrm{e}} \sigma^{2},
$$

where $D_{\mathrm{e}}$ is effective population density, which can be conveniently approximated as $D_{e}=D \times N_{e} / N$. Following the approach suggested by Vekemans and Hardy (2004), we estimated the ratio $N_{\mathrm{e}} / N$ from Equation 3 and used this estimation to calculate $D_{\mathrm{e}}$

$$
\left.N_{e} / N \equiv 4 /\left[2\left(1-F_{I}\right)+\left(1+F_{I}\right) V\right)\right],
$$

where $V$ corresponds to the variance of lifetime reproductive success among individuals (Kimura and Crow, 1963; see Appendix 2 in Supplementary Information for further details).

In populations where we detected significant FSGS consistent with IBD, we computed $\mathrm{Nb}$ (Equation 1) and used estimated values of $D_{\mathrm{e}}$ to compute $\sigma_{\mathrm{e}}$ (Equation 2), applying the iterative procedure implemented in SPAGeDi.

Approximate $95 \%$ CIs around $\mathrm{Nb}$ estimates were computed as $\left(F_{1}-1\right) /$ $\left(b_{r}+2\right.$ s.e. $\left._{\mathrm{b}}\right)$ and $\left(F_{1}-1\right) /\left(b_{r}-2\right.$ s.e. $\left._{\mathrm{b}}\right)$, respectively, where s.e.b is the standard error of $b_{\mathrm{r}}$ estimated by jackknifing over loci. When $b_{\mathrm{r}}>2$ s.e., the upper bound was reported as infinite (Fenster et al., 2003; Hardy et al., 2006). The 95\% CI of $\sigma_{\mathrm{e}}$ was obtained using an analogous procedure.

Following Crawford (1984), we decomposed $\sigma_{\mathrm{e}}$ into effective seed $\left(\sigma_{\mathrm{e}, \mathrm{s}}\right)$ and pollen $\left(\sigma_{\mathrm{e}, \mathrm{p}}\right)$ gene dispersal rates:

$$
\sigma_{\mathrm{e}}^{2}=\sigma_{e, s}^{2}+\frac{1}{2} \sigma_{e, p}^{2}
$$

We used contemporary (Crawford, 1984) field estimates of mother-seedling axial square dispersal distance $\left(\sigma_{\mathrm{s}}\right)$ obtained through inverse modelling (Lara-Romero et al., 2014) as a rough approximation of $\sigma_{\mathrm{e}, \mathrm{s}}$. We then used estimated $\sigma_{\mathrm{e}}$ and $\sigma_{\mathrm{e}, \mathrm{s}}$ values to approximate the value of $\sigma_{\mathrm{e}, \mathrm{p}}$ using Equation 4.

\section{Association between demographic structure, microhabitat heterogeneity and FSGS}

$\mathrm{O}(r)$ shares the ring-based approach with spatial genetic autocorrelation, making the results of our spatial demographic and genetic analyses directly comparable (Chung et al., 2007, 2011). Hence, to quantify the strength of the association between individual spatial aggregation and FSGS, we conducted a correlation analysis between $b_{\mathrm{Ld}}$ and $b_{\mathrm{LO}(\mathrm{r})}$ (Chung et al., 2007). We also assessed the correlation between $S p$ and adult density $(D)$ and the heterogeneity of suitable microhabitat $(S I)$. 


\section{RESULTS}

Spatial distribution of adult plants within populations

The O-ring statistic $\mathrm{O}(r)$ showed significant individual spatial aggregation at small scales in all of the studied populations (Figure 1b). PEN showed the strongest overall aggregation, with a slope of the O-ring statistic of $b_{\mathrm{LO}(\mathrm{r})}=-4.73$ (95\% CI: $\left.-5.22,-4.24\right)$. LAG and NEV exhibited intermediate patterns $\left(b_{\mathrm{LO}(\mathrm{r})}=-2.28,95 \% \mathrm{CI}:-2.71\right.$, -1.85 and $b_{\mathrm{LO}(\mathrm{r})}=-2.19,95 \% \mathrm{CI}:-2.35,-2.03$, respectively), whereas $\mathrm{CAB}$ and Najarra (NAJ) showed the flattest functions $\left(b_{\mathrm{LO}(\mathrm{r})}=-1.73\right.$, 95\% CI: $-1.87,-1.59$ and $b_{\mathrm{LO}(\mathrm{r})}=-1.18,95 \% \mathrm{CI}:-1.30,-1.06$, respectively). Pairwise $t$-tests showed significant differences in $b_{\mathrm{LO}(\mathrm{r})}$ values between all studied population pairs except between NEV and LAG (Supplementary Information and Supplementary Table S1).

\section{Population genetic analyses}

We scored a total of 144 alleles across 8 loci in 474 individuals (Table 1$)$, with a mean $( \pm$ s.e. $)$ number of $10( \pm 0.8)$ alleles per locus. All five populations contained similar levels of genetic diversity and inbreeding (Table 1). All populations showed significant departures from Hardy-Weinberg equilibrium towards heterozygote deficiency, according to global tests across loci. Detailed genetic diversity estimates for each marker and population are provided as Supplementary Information (Supplementary Table S2).

\section{Characterization of fine-scale genetic structure}

All five independent runs of Geneland gave a single genetic group for each population, indicating that there were no genetic discontinuities or spatially structured gene pools within populations.

No significant relationship was found between genetic kinship $\left(F_{\mathrm{ij}}\right)$ and geographic distance between individuals in CAB and NAJ $\left(b_{\mathrm{L}}\right.$ and $b_{\mathrm{Ld}}$ were not statistically different from 0 , Figure $1 \mathrm{c}$ and Supplementary Table S3). However, the slopes of the linear regressions between $F_{\mathrm{ij}}$ and both geographic distance and the logarithm of geographic distance were significantly negative in PEN, NEV and LAG (Figure 1c and Supplementary Table S3), as expected under IBD. In all three populations, the decrease in $F_{i j}$ with distance fitted a logarithmic relationship better than a linear relationship $\left(R_{\mathrm{dL}}^{2}>R_{\mathrm{d}}^{2}\right)$ (Supplementary Table S3), as expected for two-dimensional populations. The three populations with significant IBD showed significantly positive $F_{\mathrm{ij}}$ values in the first distance class $(d \leq 0.5 \mathrm{~m})$, rapidly declining and becoming nonsignificant in the second or third distance classes (Figure 1c). The $S p$ statistic confirmed the existence of among-population variation in FSGS intensity (Figure $1 \mathrm{c}$ and Supplementary Table S3). PEN showed the strongest FSGS with $S p=0.0161$ (95\% CI: 0.0127, 0.0221), followed by LAG and NEV, $S p=0.0117(0.029,0.0205)$ and $S p=0.0096(0.0033,0.0159)$, respectively. The lowest $S p$ values were found in NAJ and $\mathrm{CAB}$ with $S p=0.0064(0.0015,0.0113)$ and $S p=0.0009(0,0.0054)$, respectively. Pairwise $t$-tests indicated that $S p$ at PEN was significantly greater than at NAJ and $\mathrm{CAB}(t=2.27, P<0.05$ and $t=2.70, P<0.01$; respectively).

Wright's neighbourhood size $(\mathrm{Nb})$ and effective gene dispersal rate $\left(\sigma_{\mathrm{e}}\right)$ were assessed in the three populations that had a FSGS consistent with IBD. Estimates of neighbourhood size were $N b=158$ individuals (95\% CI: 121, 195) for PEN, $N b=103$ individuals $(91, \infty)$ for LAG and $\mathrm{Nb}=106$ individuals $(81, \infty)$ for NEV. $\mathrm{Nb}$ values for PEN were $\sim 10 \%$ of the total number of individuals in the plot, whereas in NEV and LAG they accounted for $\sim 40 \%$ and $60 \%$ of total plot size, respectively. The ratio of effective to census population sizes $\left(N_{\mathrm{e}} / N\right)$ was estimated at $0.279,0.265,0.268,0.272$ and 0.261 , in PEN, CAB, NAJ, NEV and LAG, respectively. These $N_{\mathrm{e}} / N$ ratios yielded effective densities $D_{\mathrm{e}}$ ranging from 0.40 to $3.57 \mathrm{ind}^{-2}$ (Table 1). Using these
$D_{\mathrm{e}}$ estimates, we obtained a notably lower $\sigma_{\mathrm{e}}$ estimate for PEN $(1.94 \mathrm{~m}, 95 \%$ CI: $1.74,2.14)$ than for NEV $\left(\sigma_{\mathrm{e}}=3.9 \mathrm{~m}, 95 \%\right.$ CI: 3.69 , $\infty)$ and LAG $\left(\sigma_{\mathrm{e}}=4.65 \mathrm{~m}, 95 \% \mathrm{CI}: 4.17, \infty\right)$. The decomposition of $\sigma_{\mathrm{e}}$ into effective seed $\left(\sigma_{\mathrm{e}, \mathrm{s}}\right)$ and pollen $\left(\sigma_{\mathrm{e}, \mathrm{p}}\right)$ gene dispersal rates showed that among-population differences in $\sigma_{\mathrm{e}}$ seemed to be mainly mediated by differences in pollen-mediated gene dispersal. In particular, $\sigma_{\mathrm{e}, \mathrm{s}}$ estimates were similar in all populations $(0.27,0.21$ and $0.20 \mathrm{~m}$ for PEN, NEV and LAG, respectively), whereas the estimate of $\sigma_{\mathrm{e}, \mathrm{p}}$ for PEN was $1.35 \mathrm{~m}$, less than half of those for NEV and LAG (2.75 and $3.28 \mathrm{~m}$, respectively).

\section{Association between demographic structure, microhabitat heterogeneity and FSGS}

We found a significant strong association between spatial autocorrelation measures of genetic structure $\left(b_{\mathrm{Ld}}\right)$ and individual spatial aggregation $\left(b_{\mathrm{LO}(\mathrm{r})}\right)$ (Figure 2; Pearson's product-moment correlation: $r=0.93, P=0.02)$. The correlation between $S p$ and microhabitat heterogeneity $(S I)$ was high but not significant (Figure 3b: $r=0.69$, $P=0.20)$. No correlation was found between $S p$ and adult density $(D)$ (Figure 3a; $r=-0.15, P=0.81$ ).

\section{DISCUSSION}

This study found significant FSGS, consistent with IBD, in three of the five studied populations of $S$. ciliata, as well as significant among-population variation in the intensity of FSGS. Spatial aggregation of individuals and FSGS were positively correlated, supporting the influence of fine-scale aggregation of plants on intraspecific FSGS variation. The populations that did not exhibit significant IBD corresponded to those with the lowest spatial aggregation. On the other hand, FSGS was not significantly correlated with global population density or microhabitat heterogeneity.

Assuming dispersal-drift equilibrium, the observed differences in FSGS among the studied populations, as measured by $S p$, should mainly result from differences in the product between effective gene dispersal and effective population density (that is, the genetic neighbourhood size, inversely related to Sp: Wright, 1943; Rousset, 2000; Vekemans and Hardy, 2004). Effective gene dispersal $\sigma_{\mathrm{e}}$ is defined as the (long-term) asymptotic mean-square dispersal distance of gene lineages across generations, akin to a diffusion rate of genes in the landscape (Wright, 1943; Rousset, 2000; Vekemans and Hardy, 2004). Thus, the magnitude of $\sigma_{\mathrm{e}}$ and its ensuing effects on $S p$ depend

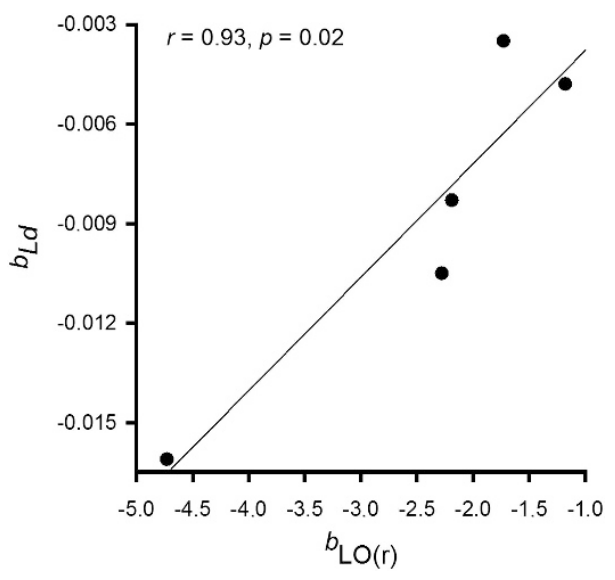

Figure 2 Association between spatial autocorrelation measures of fine-scale genetic structure $\left(b_{\mathrm{Ld}}\right)$ and individual spatial aggregation $\left(b_{\mathrm{LO}(\mathrm{r})}\right)$; ' $r$ ' indicates Pearson's product-moment correlation. 

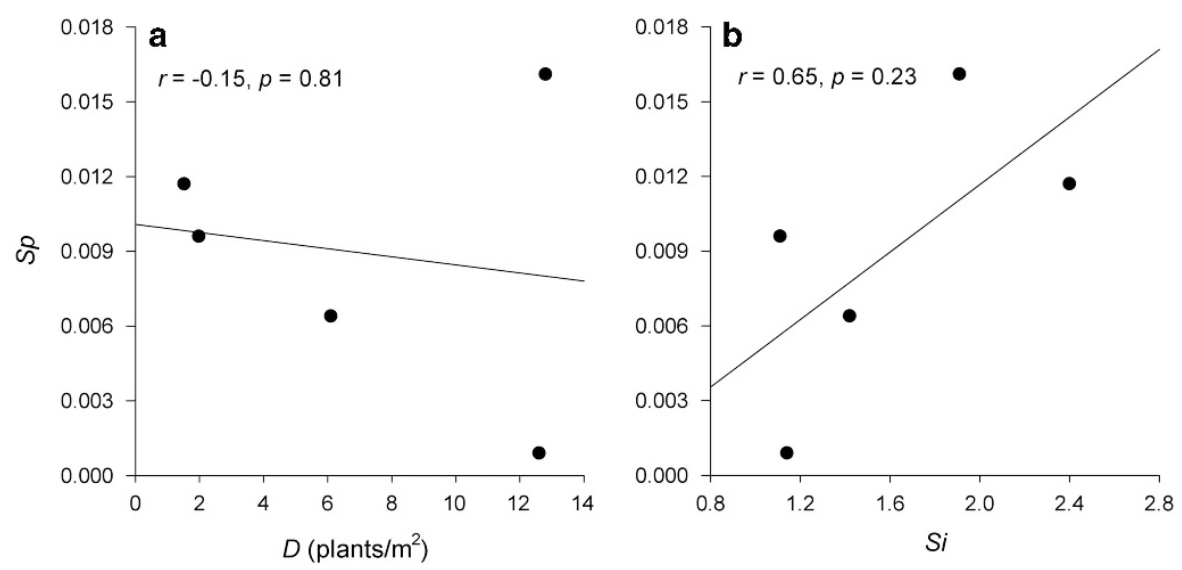

Figure 3 Association between the fine-scale spatial genetic structure $(S p)$ and (a) adult density $(D)$ and (b) microhabitat heterogeneity $(S /)$; ' $r$ ' indicates Pearson's product-moment correlation.

on both the dispersal rate over a single generation $(\sigma)$ and the permeability of the landscape to the long-term diffusion of gene lineages (Robledo-Arnuncio and Rousset, 2010). If favourable habitat is not continuous, and especially if the discontinuities remain static across generations, some gene lineages may remain on the edges of a favourable habitat patch over several generations until an interpatch dispersal event occurs. This resistance to gene diffusion may result in $\sigma_{\mathrm{e}}$ values substantially lower than $\sigma$ (Robledo-Arnuncio and Rousset, 2010). Furthermore, $\sigma$ itself may be lower in spatially aggregated populations, if plant clustering reduces seed or pollen dispersal vector mobility, for instance if the proportion of intrapatch pollinator flights tends to increase with spatial aggregation (Morris, 1993; Cresswell and Osborne, 2004). Overall, FSGS is expected to be stronger in spatially aggregated populations than in continuous populations, even if, on average, $\sigma$ remains unchanged.

Consistent with this prediction, we found a positive correlation between the spatial aggregation of individuals and FSGS, as well as the lowest value of $\sigma_{\mathrm{e}}$ estimated for the population with the strongest individual spatial aggregation and FSGS. This suggests that variation in the spatial distribution of individuals generates intraspecific variation in FSGS for the study species, even if average seed dispersal distances are largely invariant across populations (Lara-Romero et al., 2014). The positive correlation between spatial aggregation and FSGS would not be expected in plant species or populations where the scale of seed and/or pollen dispersal was large relative to the distance between favourable patches, nor if individual aggregation resulted from habitat heterogeneities that change rapidly over generations (Robledo-Arnuncio and Rousset, 2010). In future, it would be of interest to empirically assess potential associations between spatial and genetic population structure as a function of the dispersal ability of species and temporal habitat dynamics.

Although previous empirical studies have suggested that spatial heterogeneity could be a potential determinant of variation in FSGS (Valbuena-Carabaña et al., 2007; Born et al., 2008; Wang et al., 2011), its association with spatial aggregation and its ensuing effects on effective gene dispersal rates have rarely been measured. The few studies that aimed to quantify both the local distribution of individuals and genetic structure across plant populations found positive associations between the level of FSGS and spatial aggregation (Jacquemyn et al., 2009; Chung et al., 2011). However, these studies compared continuous stable populations with expanding and senescing spatially aggregated populations, or seedlings to adult plants within the same population. As far as we know, this is the first empirical study reporting evidence of a possible relationship between the spatial aggregation of plants and FSGS in equilibrium populations.

Contrary to our expectations, the correlation between the intensity of FSGS and global population density was not significant. This could have resulted from the low statistical power of the design because, with only 5 populations, an observed value of $r>0.81$ is required for statistical significance at the $5 \%$ level. If PEN population is considered an outlier and removed from the analysis (for example, on the basis of having experienced a different demographic history than the rest of the populations), then there is a clear trend of decreasing $S p$ with $D$, as the theory predicts (Leblois et al., 2004; Vekemans and Hardy, 2004). However, our results suggest a common history of all studied populations. Rather than reflecting a statistical issue, the lack of a significant association between $S p$ and global population density could also be explained by at least two different biological processes. First, it could result from the interacting effects of $D_{\mathrm{e}}$ and spatial aggregation on FSGS. Indeed, even if increasing $D_{\mathrm{e}}$ is expected to decrease FSGS, this effect can be masked by the opposite (positive) effect of a high level of spatial aggregation on FSGS. This is likely the case in this study, because both $D_{e}$ and the levels of spatial aggregation vary among populations. Such an effect could explain why the two populations with the highest, similar, densities (the weakly aggregated population $\mathrm{CAB}$ and the strongly aggregated PEN) exhibited contrasting levels of FSGS (with larger $S p$ in PEN). Second, pollinator behaviour could invert the direction of the relationship between $D_{\mathrm{e}}$ and FSGS. Namely, global density is assumed to cause a reduction in FSGS through decreased gene identity probabilities; however if pollinators flew longer distances (that is, increased $\sigma_{\mathrm{e}}$ ) in low- than in high-density populations (as found in several empirical studies, see, for example, Tero et al., 2005; Barluenga et al., 2011), this effect could result in stronger genetic structure in high-density populations. This effect could explain why $S p$ was not significantly different in high-density PEN than in low-density LAG and NEV. It is also consistent with the inferred effective pollen gene dispersal rate $\sigma_{\mathrm{e}, \mathrm{p}}$ that was less than half of the values calculated for NEV and LAG (2.75 and $3.28 \mathrm{~m}$, respectively) in PEN (1.35 m).

\section{CONCLUSIONS}

This study shows that variation in the fine-scale spatial distributions of individuals can be associated with intraspecific variation in FSGS, even if average seed dispersal distances are invariant across populations. These findings support theoretical studies indicating that stronger individual spatial aggregation generally increases the magnitude of 
FSGS (Doligez et al., 1998; Barton and Keightley, 2002; RobledoArnuncio and Rousset, 2010). No linear association was found between global plant density, or microhabitat heterogeneity, and FSGS. These results are difficult to interpret because of the low statistical power of our analysis; however, they suggest that individual spatial aggregation is more relevant than global plant density and microhabitat heterogeneity in determining variation in FSGS among $S$. ciliata populations. Additional research is required to confirm our findings and to evaluate their generality in a wider range of taxa and environments. This study highlights the importance of combining spatial analyses of genotypes, individuals and microhabitats to better understand the mechanisms generating intraspecific variation in FSGS across landscapes.

\section{DATA ARCHIVING}

The data sets used for this study including the location of each $S$. ciliata adult plant within the study plots and microsatellite data are available at the institutional repository of Rey Juan Carlos University (BURJC-Digital, http://eciencia.urjc.es/handle/10115/12500).

\section{CONFLICT OF INTEREST}

The authors declare no conflict of interest.

\section{ACKNOWLEDGEMENTS}

We thank Alex Widmer (ETH-Zurich) for his valuable comments on the manuscript and Lori De Hond for linguistic assistance. We also thank Marcelino de la Cruz (ESCET, Universidad Rey Juan Carlos) for his helpful recommendations for analysing spatial point pattern data and the staff of Sierra de Guadarrama National Park for permission to work in the field area. This work was supported by the project AdAptA (CGL2012-33528). CL-R and JM-L were supported by a FPI fellowship (Spanish Ministry of Science).

Baddeley A, Turner R (2005). Spatstat: an R package for analyzing spatial point patterns. J Stat Softw 12: 1-42.

Barluenga M, Austerlitz F, Elzinga JA, Teixeira S, Goudet J, Bernasconi G (2011). Fine-scale spatial genetic structure and gene dispersal in Silene latifolia. Heredity 106: 13-24.

Barton NH, Keightley PD (2002). Understanding quantitative genetic variation. Nat Rev Genet 3: 11-21.

Berry JK (2007). Map Analysis: Understanding Spatial Patterns and Relationships. GeoTec Media: Los Angeles, CA

Born C, Hardy OJ, Chevallier M-H, Ossari S, Atteke C, Wickings E et al. (2008). Small-scale spatial genetic structure in the Central African rainforest tree species Aucoumea klaineana: a stepwise approach to infer the impact of limited gene dispersal, population history and habitat fragmentation. Mol Ecol 17: 2041-2050.

Chung MY, Nason JD, Chung MG (2007). Effects of population succession on demographic and genetic processes: predictions and tests in the daylily Hemerocallis thunbergii (Liliaceae). Mol Ecol 16: 2816-2829.

Chung MY, Nason JD, Chung MG (2011). Significant demographic and fine-scale genetic structure in expanding and senescing populations of the terrestrial orchid Cymbidium goeringii (Orchidaceae). Am J Bot 98: 2027-2039.

Crawford TJ (1984). The estimation on neighbourhood parameters for plant populations. Heredity 52: 273-283.

Cresswell JE, Osborne JL (2004). The effect of patch size and separation on bumblebee foraging in oilseed rape: implications for gene flow. J Appl Ecol 41: 539-546.

Doligez A, Baril C, Joly HI (1998). Fine-scale spatial genetic structure with nonuniform distribution of individuals. Genetics 148: 905-919.
Ennos RA (1994). Estimating the relative rates of pollen and seed migration among plant populations. Heredity 72: 250-259.

Fenster CB, Vekemans X, Hardy OJ (2003). Quantifying gene flow from spatial genetic structure data in a metapopulation of Chamaecrista fasciculata (Leguminosae). Evolution 57: 995-1007.

García-Fernández A, Segarra-Moragues JG, Widmer A, Escudero A, Iriondo JM (2012). Unravelling genetics at the top: mountain islands or isolated belts? Ann Bot 110 1221-1232.

Gimenez-Benavides L, Escudero A, Iriondo JM (2007). Reproductive limits of a lateflowering high-mountain Mediterranean plant along an elevational climate gradient. New Phytol 173: 367-382.

Gimenez-Benavides L, Escudero A, Iriondo JM (2008). What shapes the altitudinal range of a high mountain Mediterranean plant? Recruitment probabilities from ovule to seedling stage. Ecography 31: 731-740.

Guillot G, Mortier F, Estoup A (2005). GENELAND: a computer package for landscape genetics. Mol Ecol Notes 5: 712-715.

Hamrick JL, Trapnell DW (2011). Using population genetic analyses to understand seed dispersal patterns. Acta Oecologica 37: 641-649.

Hardy OJ, Maggia L, Bandou E, Breyne P, Caron H, Chevallier M et al. (2006). Fine-scale genetic structure and gene dispersal inferences in 10 Neotropical tree species. $\mathrm{Mol}$ Ecol 15: 559-571.

Hardy OJ, Vekemans X (2002). SPAGeDi: a versatile computer program to analyse spatial genetic structure at the individual or population levels. Mol Ecol Notes 2: 618-620.

Jacquemyn H, Wiegand T, Vandepitte K, Brys R, Roldán-Ruiz I, Honnay O (2009). Multigenerational analysis of spatial structure in the terrestrial, food-deceptive orchid Orchis mascula. J Ecol 97: 206-216.

Kimura M, Crow JF (1963). The measurement of effective population number. Evolution 17: 279-288.

Lara-Romero C, Robledo-Arnuncio JJ, García-Fernández A, Iriondo JM (2014). Assessing intraspecific variation in effective dispersal along an altitudinal gradient: a test in two Mediterranean high-mountain plants. PLoS One 9: e87189.

Leblois R, Estoup A, Streiff R (2006). Genetics of recent habitat contraction and reduction in population size: does isolation by distance matter? $\mathrm{Mol}$ Ecol 15 3601-3615.

Leblois R, Rousset F, Estoup A (2004). Influence of spatial and temporal heterogeneities on the estimation of demographic parameters in a continuous population using individual microsatellite data. Genetics 166: 1081-1092.

Linhart YB, Grant MC (1996). Evolutionary significance of local genetic differentiation in plants. Annu Rev Ecol Syst 27: 237-277.

Linhart YB, Mitton JB, Sturgeon KB, Davis ML (1981). Genetic variation in space and time in a population of ponderosa pine. Heredity 46: 407-426.

Loiselle BA, Sork VL, Nason J, Graham C (1995). Spatial genetic structure of a tropical understory shrub, Psychotria officinalis (Rubiaceae). Am J Bot 82: 1420-1425.

Morris WF (1993). Predicting the consequences of plant spacing and biased movement for pollen dispersal by honey bees. Ecology 74: 493-500.

Robledo-Arnuncio JJ, Rousset F (2010). Isolation by distance in a continuous population under stochastic demographic fluctuations. J Evol Biol 23: 53-71.

Rousset F (2000). Genetic differentiation between individuals. J Evol Biol 13 (1): 58-62.

Storfer A, Murphy MA, Evans JS, Goldberg CS, Robinson S, Spear SF et al. (2007). Putting the 'landscape' in landscape genetics. Heredity 98: 128-142.

Tero N, Aspi J, Siikamäki P, Jäkäläniemi A (2005). Local genetic population structure in an endangered plant species, Silene tatarica (Caryophyllaceae). Heredity 94: 478-487.

Valbuena-Carabaña M, González-Martínez SC, Hardy OJ, Gil L (2007). Fine-scale spatial genetic structure in mixed oak stands with different levels of hybridization. $\mathrm{Mol} \mathrm{Ecol} 16$ : 1207-1219.

Van Heerwaarden J, Ross-Ibarra J, Doebley J, Glaubitz JC, Sánchez-González JJ, Gaut BS, Eguiarte LE (2010). Fine scale genetic structure in the wild ancestor of maize (Zea mays ssp. parviglumis). Mol Ecol 19: 1162-1173.

Van Oosterhout C, Hutchinson WF, Wills DPM, Shipley P (2004). MICRO-CHECKER: software for identifying and correcting genotyping errors in microsatellite data. $\mathrm{Mol} E \mathrm{CO}$ Notes 4: 535-538.

Vekemans X, Hardy OJ (2004). New insights from fine-scale spatial genetic structure analyses in plant populations. $\mathrm{Mol} \mathrm{ECOl}$ 13: 921-935.

Wang R, Compton SG, Chen X-Y (2011). Fragmentation can increase spatial genetic structure without decreasing pollen-mediated gene flow in a wind-pollinated tree. $\mathrm{Mol}$ Ecol 20: 4421-4432.

Wiegand T, Moloney KA (2004). Rings, circles, and null-models for point pattern analysis in ecology. Oikos 104: 209-229.

Wright S (1943). Isolation by distance. Genetics 28: 114

Supplementary Information accompanies this paper on Heredity website (http://www.nature.com/hdy) 